\title{
Patterns of mitochondrial DNA instability in Brassica campestris cultured cells
}

\author{
Magid Shirzadegan ${ }^{1,3}$, Jeffrey D. Palmer ${ }^{1,4}$, Mary Christey ${ }^{2,5}$ and Elizabeth D. Earle ${ }^{2}$ \\ ${ }^{1}$ Department of Biology, University of Michigan, Ann Arbor, MI 48109, USA ; ${ }^{2}$ Department of Plant \\ Breeding, Cornell University, Ithaca, NY 14853-1902, USA; Present addresses: ${ }^{3}$ Department of \\ Biochemistry, University of Nevada, Reno, NV 89557, USA ; ${ }^{4}$ Department of Biology, Indiana University, \\ Bloomington, IN 47405, USA ; ${ }^{5}$ Crop Research Division, DSIR, Lincoln, New Zealand
}

Received 2 May 1990; accepted in revised form 11 September 1990

Key words: Brassica, cultured cells, mitochondrial DNA, mitochondrial DNA instability, heterogeneity, sublimon

\begin{abstract}
We previously showed that the mitochondrial DNA (mtDNA) of a Brassica campestris callus culture had undergone extensive rearrangements (i.e. large inversions and a duplication) relative to DNA of the control plant [54]. In this study we observed that after continued growth, the mtDNA of this culture continues to change, with rearranged forms amplifying and diminishing to varying proportions. Strikingly similar changes were detected in the mtDNA profiles of a variety of other long- and short-term callus and cell suspension lines. However, the proportions of parental ('unrearranged') and novel ('rearranged') forms varied in different cultured cell mtDNAs. To address the source of this heterogeneity, we compared the mtDNA organization of 28 individual plants from the parental seed stock. With the exception of one plant containing high levels of a novel plasmid-like mtDNA molecule, no significant variation was detected among individual plants and therefore source plant variation is unlikely to have contributed to the diversity of mitochondrial genomes observed in cultured cells. The source of this culture-induced heterogeneity was also investigated in 16 clones derived from single protoplasts. A mixed population of unrearranged and rearranged mtDNA molecules was apparent in each protoclone, suggesting that the observed heterogeneity in various cultures might reflect the genomic composition of each individual cell; however, the induction of an intercellular heterogeneity subsequent to the protoplast isolation was not tested and therefore cannot be ruled out. The results of this study support our earlier model that the rapid structural alteration of $B$. campestris mtDNA in vitro results from preferential amplification and reassortment of minor pre-existing forms of the genome rather than de novo rearrangement. Infrequent recombination between short dispersed repeated elements is proposed as the underlying mechanism for the formation of these minor mtDNA molecules.
\end{abstract}

\section{Introduction}

In vitro culture of plant cells has become an increasingly important tool in genetic manipu- lation and improvement of major crops [for review see 3]. Broader application of tissue culture technology, however, has been limited to some degree by the accompanying somaclonal 
variation often observed in the nuclear and organellar genomes of many plant species [for review see 22]. Although the majority of these genetic mutations have been attributed to the nucleus [26, 14], recent molecular analyses of plant mitochondrial DNA (mtDNA) indicate that when plant tissues are subjected to conditions of prolonged culture, extensive structural changes (i.e. inversion, deletion/insertion, transposition) in the organization of the mitochondrial genome may be observed $[7,34,62]$. The instability of the mitochondrial genome has been investigated in somatic hybrids $[15,38,60,41,21]$ as well as in vitro cultures of various plant systems $[8,39,18,58,50$, 4]. An often cited example of culture-induced somaclonal variation associated with mtDNA is the reversion to fertility of different cytoplasmic malesterile types in maize $[16,5,12,48]$. In several reports, the nature of mitochondrial genome alteration in vitro has been attributed to the culture system, such as the age [34], culture type [7], or the in vitro technique employed [23].

We recently reported that the mitochondrial genome of a 29-month-old Brassica campestris cell culture was extensively rearranged and included molecules containing at least two inversions and one large duplication [54]. This finding raises questions as to whether the same or different pattern of rearrangement will occur in other culture lines and to how the novel forms of the genome arise. To address these questions, we have examined the mtDNA of the original callus culture line at approximately six-month intervals for two additional years and have compared the mitochondrial genome organization of other independently established cell lines. Furthermore, the mtDNA profiles of 28 individual $B$. campestris plants and 16 clones derived from individual protoplasts from the original callus were examined to address the source of the heterogeneity detected in the mitochondrial genome of the cultured cells. We discuss the role of recombination at short repeated elements as the underlying mechanism for the rapid structural alterations of mtDNA and provide evidence that culture-induced mtDNA alterations result primarily from the differential amplification of pre-existing rearranged genome forms rather than from de novo rearrangement.

\section{Materials and methods}

The rapid-cycling, self-compatible Brassica campestris L. (CrGC 66) from which most cultures were established was the main source of the plant genomic DNA used in this study. As sources for control plant mtDNA, we utilized two different turnip varieties, 'Torch' and 'Tobin', kindly provided by Dr. Rakow at Agriculture Canada. With the exception of one culture (X), which was initiated from an atrazine-resistant line (cv. 'Candle'), all of the B. campestris cultures utilized in this investigation were established from the rapid-cycling line, CrGC 66 (Table 1). Friable and compact callus cultures as well as suspension cell lines were initiated and maintained as described previously $[27,54]$. Protoclones were recovered from the 43-month-old callus culture of BCS-A using the procedure described by Lentini et al. [27] and Robertson and Earle [49].

Plant mitochondrial and total DNA samples were prepared from fresh leaves as well as flower and stem tissues of one- to two-month-old plants. Total DNAs from both the whole plant and the cultured cells were isolated using the procedure described by Doyle and Doyle [10] slightly modified in our laboratory. Whole plant and culture mtDNAs were isolated by the DNase-I procedure of Kolodner and Tewari [25], followed by $\mathrm{CsCl}$ centrifugation, isopropanol extraction and dialysis [43]. Restriction endonuclease digestion, agarose gel electrophoreses, bidirectional tranfers of DNA fragments from agarose gels to Zetabind (AMF Cuono) membrane, labeling of recombinant plasmids by nick-translation, and filter hybridizations were performed as described earlier [54]. Recombinant plasmids containing $B$. campestris mtDNA fragments came from the library described by Palmer and Shields [44].

\section{Results}

$m t D N A$ instability in vitro over time

In our previous study, we compared $B$. campestris mitochondrial DNA (mtDNA) from whole plants and from a 29-month-old cell culture [54]. 
Table 1. Brassica campestris L. cultures used in this study

\begin{tabular}{|c|c|c|c|c|}
\hline Designation & $\begin{array}{l}\text { Type of } \\
\text { Culture }\end{array}$ & Source & $\begin{array}{l}\text { Months in } \\
\text { Culture* }^{*}\end{array}$ & Figures \\
\hline BCS-A** & friable callus & hypocotyl (CrGC 66) & 29 & 1,2 \\
\hline BCS-A1 & friable callus & BCS-A & 37 & $3,4,5$ \\
\hline $\mathrm{BCS}-\mathrm{A} 2$ & friable callus & BCS-A & 44 & $3,6,7,8$ \\
\hline $\mathrm{BCS}-\mathrm{A} 3$ & friable callus & BCS-A & 53 & $2,3,4$ \\
\hline BCS-B I & compact callus & hypocotyl (CrGC 66) & 8 & $5,6,7$ \\
\hline BCS-B2 & compact callus & BCS-B 1 & 17 & 6 \\
\hline $\mathrm{BCS}-\mathrm{Cl}$ & compact callus & hypocotyl (CrGC 66) & 6 & 5,6 \\
\hline $\mathrm{BCS}-\mathrm{C} 2$ & friable callus & BCS-C1 & 15 & 6 \\
\hline BCS-C3 & friable callus & BCS-Cl & 16 & 6 \\
\hline G & friable callus & hypocotyl (CrGC 66) & 11 & 5 \\
\hline $\mathrm{K}$ & friable callus & hypocotyl (CrGC 66) & 11 & 5 \\
\hline $\mathbf{L}$ & friable callus & hypocotyl (CrGC 66) & 11 & 5 \\
\hline $\mathbf{M}$ & friable callus & hypocotyl (CrGC 66) & 11 & 5 \\
\hline $\mathrm{Q}$ & friable callus & hypocotyl (CrGC 66) & 11 & 5 \\
\hline $\mathrm{X}$ & friable callus & hypocotyl (cv. Candle) & 11 & 5 \\
\hline BCS-As & suspension & $\begin{array}{l}36 \text { month old } \\
\text { BCS-A callus }\end{array}$ & $\begin{array}{l}48 \text {; last } 12 \\
\text { in suspension }\end{array}$ & 7 \\
\hline BCS-Bs & suspension & $\begin{array}{l}6 \text { month old } \\
\text { BSC-B callus }\end{array}$ & $\begin{array}{l}16 \text {; last } 11 \\
\text { in suspension }\end{array}$ & 7 \\
\hline $8 \mathrm{a}$ & suspension & BCS-A callus & $\begin{array}{l}48 \text {; last } 12 \\
\text { in suspension }\end{array}$ & $8 \mathrm{~A}$ \\
\hline P-6 & friable protoclone & $\begin{array}{l}29 \text { month old } \\
\text { BCS-A callus }\end{array}$ & $\begin{array}{l}37 \text {; last } 10 \\
\text { as protoclone }\end{array}$ & $8 \mathrm{~A}$ \\
\hline $\begin{array}{l}\text { P-10, 15, 16, } \\
36,37,38,40 \\
42,43,44,45\end{array}$ & friable protoclones & $\begin{array}{l}43 \text { month old } \\
\text { BCS-A callus }\end{array}$ & $\begin{array}{l}46 \text {; last } 3 \\
\text { as protoclone }\end{array}$ & $8 \mathrm{~A}$ \\
\hline $\mathrm{H}-1, \mathrm{H}-2, \mathrm{H}-3$ & compact protoclones & $\begin{array}{l}43 \text { month old } \\
\text { BCS-A callus }\end{array}$ & $\begin{array}{l}46 \text {; last } 3 \\
\text { as protoclone }\end{array}$ & $8 \mathrm{~A}$ \\
\hline
\end{tabular}

* Age of the culture at the time of DNA analysis.

${ }^{* *}$ BSC lines A (A, A1, A2, A3), B (B1, B2) and C (C1, C2, C3) were derived independently.

Restriction pattern analysis, as well as hybridization studies utilizing a nearly complete $B$. campestris mtDNA clone library, revealed that extensive alteration of the culture mtDNA occurred during the 29 months of in vitro culture. Figure 1 illustrates several examples of these analyses. Note that each of the indicated cloned fragments (S10.1, P9.7, 12.2, P4.4, S4.4) hybridizes with variable intensity to the expected parental (plantlike) band as well as at least one novel band (R) in the culture mtDNA (Fig. 1). Low levels of some of the culture-associated rearranged fragments were also detectable in the mtDNA sample of the control plant (e.g. SalI fragment of $13.0 \mathrm{~kb}$ and $P s t \mathrm{I}$ fragment of $16.9 \mathrm{~kb}$ ). Figure 2 summarizes a tentative outline of the major rearrangement events in this 29-month-old cell culture (BCS-A). We postulated that at least 4 independent rearrangements had occurred in the mtDNA of this culture. One inversion was detected within Pst I fragments of $19.4 \mathrm{~kb}$ (encompassing the cloned fragment $\mathrm{S} 10.1$ ) and $12.2 \mathrm{~kb}$. The 

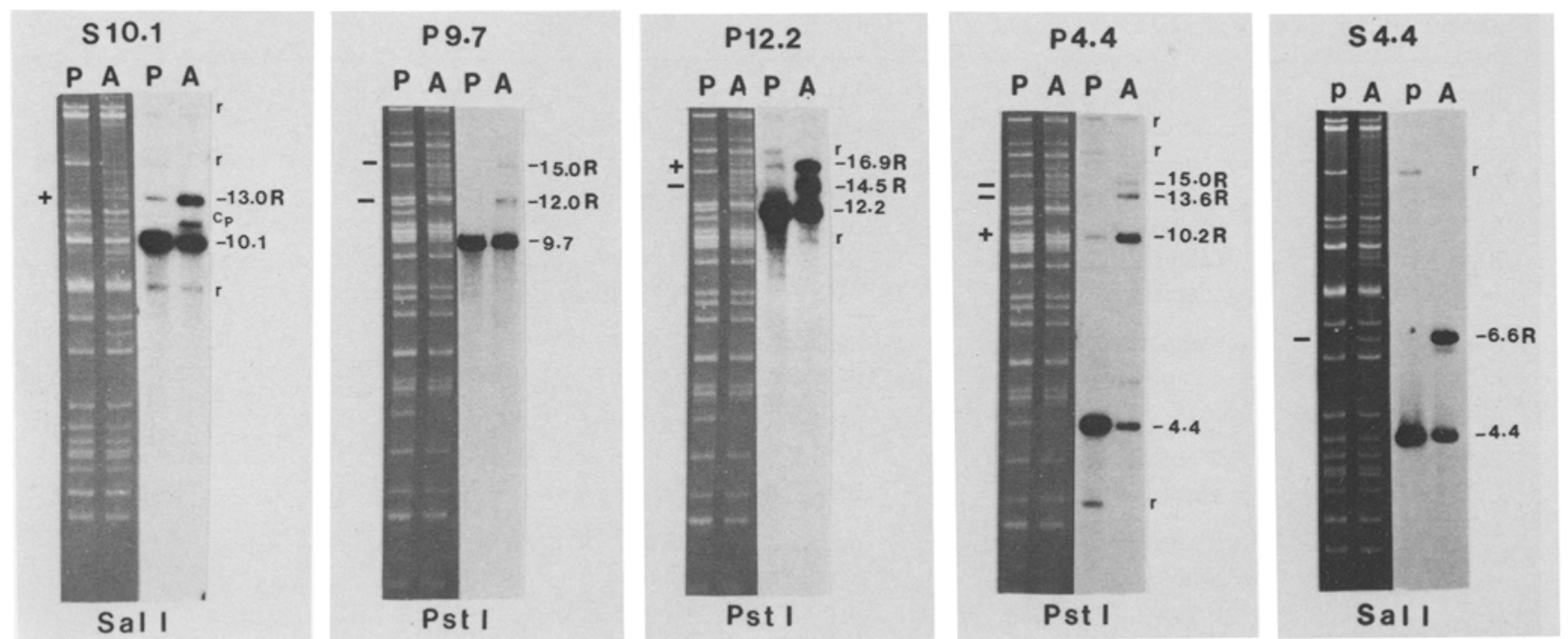

Fig. 1. Mitochondrial DNA rearrangements of Brassica campestris cultured cells. mtDNA from whole plants (P) and a 29-monthold callus culture (A) were digested with $P_{s t} \mathrm{I}$ and SalI, separated on a $0.7 \%$ agarose gel, transferred bidirectionally to Zetabind membranes, and hybridized with the indicated cloned fragments. The clone designations indicate the type of fragment cloned ( $\mathrm{S}$ for SalI and $\mathrm{P}$ for Pst $\mathrm{I}$ ) and its size in kilobase pairs. Sizes are given for parental and rearranged fragments (R) but not for small dispersed repeats ( $r$ ) or fragments resulting from chloroplast DNA contamination (Cp). The existence of low levels of culture-associated rearranged fragments in the mtDNA sample of the control plants is indicated by a plus $(+)$ sign. The minus ( - ) sign denotes the lack of a detectable level of culture-associated altered fragments in the mitochondrial genome of the control plants.

endpoints of another inversion mapped within the SalI and Pst I fragments of $4.4 \mathrm{~kb}$. However, the variable intensity of the hybridization signals of S4.4 (ca. 50\%; Fig. 1) and P4.4 (less than 10\%; Fig. 1) to their parental fragments indicated that a portion of the BCS-A mtDNA molecules (ca. $40 \%$ ) must contain a second separate rearrangement involving the $\mathrm{P} 4.4$ region (Fig. 2). Sal I fragments of $6.2 \mathrm{~kb}$ and $1.1 \mathrm{~kb}$ were also found to be duplicated in BCS-A [54]. Finally, the minor hybridization signals of P9.7 to two novel Pst I fragments of 12.0 and $15.0 \mathrm{~kb}$ suggested the occurrence of a fourth rearrangement in a small portion (ca. $10 \%$; Fig. 1) of the BCS-A mtDNA molecules.

In order to examine whether the extensive mtDNA alterations observed in the 29-month-old culture were stable, mtDNA was isolated from the BCS-A culture at subsequent seven- to ninemonth intervals and analyzed with four restriction enzymes, Pst I, SalI, BglI, and $\mathrm{Nru}$ I. In addition, a number of cloned fragments corre- sponding to the previously identified altered regions of the genome were used as hybridization probes (e.g. Figs. 3 and 4). In our previous analysis, we noted that a Pst I fragment of $4.4 \mathrm{~kb}$ was highly rearranged, containing the endpoints of at least two independent inversions (Fig. 2). Based on the low hybridization intensity of P4.4 to the parental band (Fig. 1), we had estimated that only $10 \%$ of the BCS-A mtDNA molecules contained the parental arrangement of this region (Fig. 2). As Fig. 3 illustrates, after eight months of continued growth and at both further timepoints, P4.4 and the corresponding S17.0 parental fragments are from culture mtDNA. A deletion of approximately $1.1 \mathrm{~kb}$ within P4.4 (Fig. 2) and a second putative inversion involving the $\mathrm{S} 4.4$ region (Fig. 3; note that the rearranged SalI fragments of 9.6 and $4.8 \mathrm{~kb}$ that are hybridized to in common by P4.4 and S4.4 were not present in the 29-month-old culture; Fig. 1) appear to be the primary source of the more recent changes leading to the disappearance of the Pst $\mathrm{I}$ fragment of 


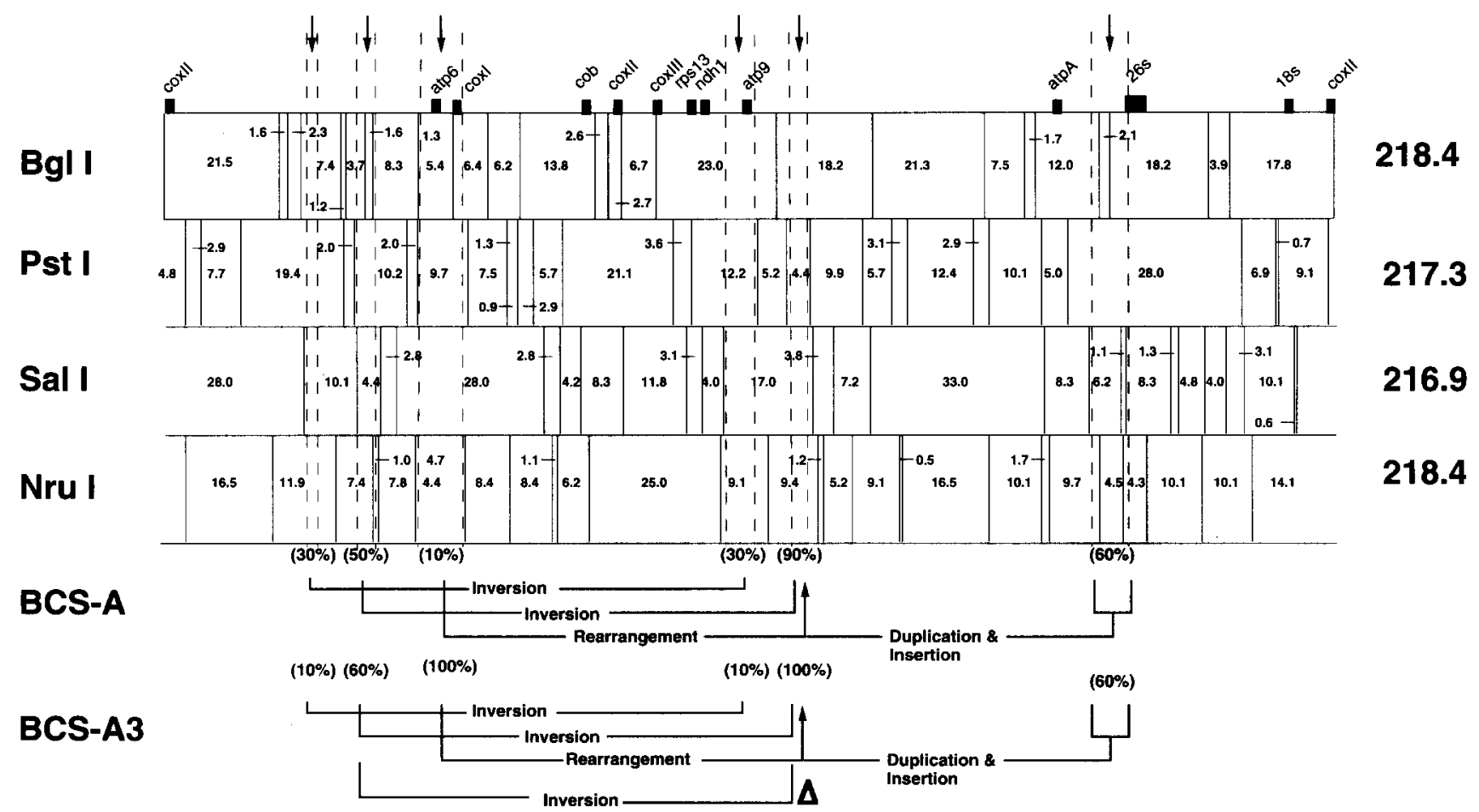

Fig.2. Locations and nature of culture-associated rearrangements in the mitochondrial genome of Brassica campestris. The circular map of the B. campestris 'master chromosome' shown linearized at the BglI site is from Palmer and Shields [44]. The summary of mtDNA rearrangements in a 29-month-old culture (BCS-A) is based on published work of Shirzadegan et al. [54; see also Fig. 1], while the summary for the 53-month-old (BCS-A3) is based on the results reported in this paper. The arrows indicate the sites, as bounded by the dashed lines of rearrangement breakpoints in the mitochondrial genome of cultured cells. A deletion of $1.1 \mathrm{~kb}$ within a Pst I fragment of $4.4 \mathrm{~kb}$ is denoted with a hollow triangle. The percentages in parentheses indicate the proportion of altered mtDNA molecules in BCS-A and BCS-A3.

\section{$4.4 \mathrm{~kb}$ and its corresponding restriction fragments} (Fig. 3).

Further evidence for continuing change of mtDNA in vitro is provided by examining P9.7, another unstable region of the Brassica mitochondrial genome (Fig. 2). Although in BCS-A, only $10 \%$ of the mtDNA molecules were altered in this region (Fig. 1), after eight additional months of in vitro growth (BCS-A1) molecules that were rearranged in the $\mathrm{P} 9.7$ region reached $50 \%$ of the mtDNA population (Fig. 4A). Furthermore, by the fifth year of subculturing (BCS-A3), we were unable to detect any traces of P9.7 and its corresponding parental fragments, SalI fragment of $28.0 \mathrm{~kb}$ and $\mathrm{Nru} \mathrm{I}$ fragment of $4.7 \mathrm{~kb}$ (Fig. 4A). Thus parental fragments P4.4 and P9.7 were both ultimately lost completely from the culture
mtDNA and replaced by a number of rearranged fragments, an indication of multiple and continuing rearrangements. However, the kinetics of genome reassortment varied for the two regions, in that P4.4 was absent after 37 months of culture (BCS-A1; Fig. 3), whereas P9.7 disappeared only after 53 months (BCS-A3; Fig. 4A).

Hybridization analysis utilizing cloned fragments that correspond to other previously identified altered regions (i.e. S10.1, P12.2, S6.2) failed to demonstrate any new alterations occurring over time within the mitochondrial genome of BCS-A (Fig. 4B and data not shown). However, quantitative variation in the stoichiometry of several novel fragments was evident. For example, culture-associated altered fragments (e.g. P14.5, P16.8, S13.0, N11.3) resulting from 


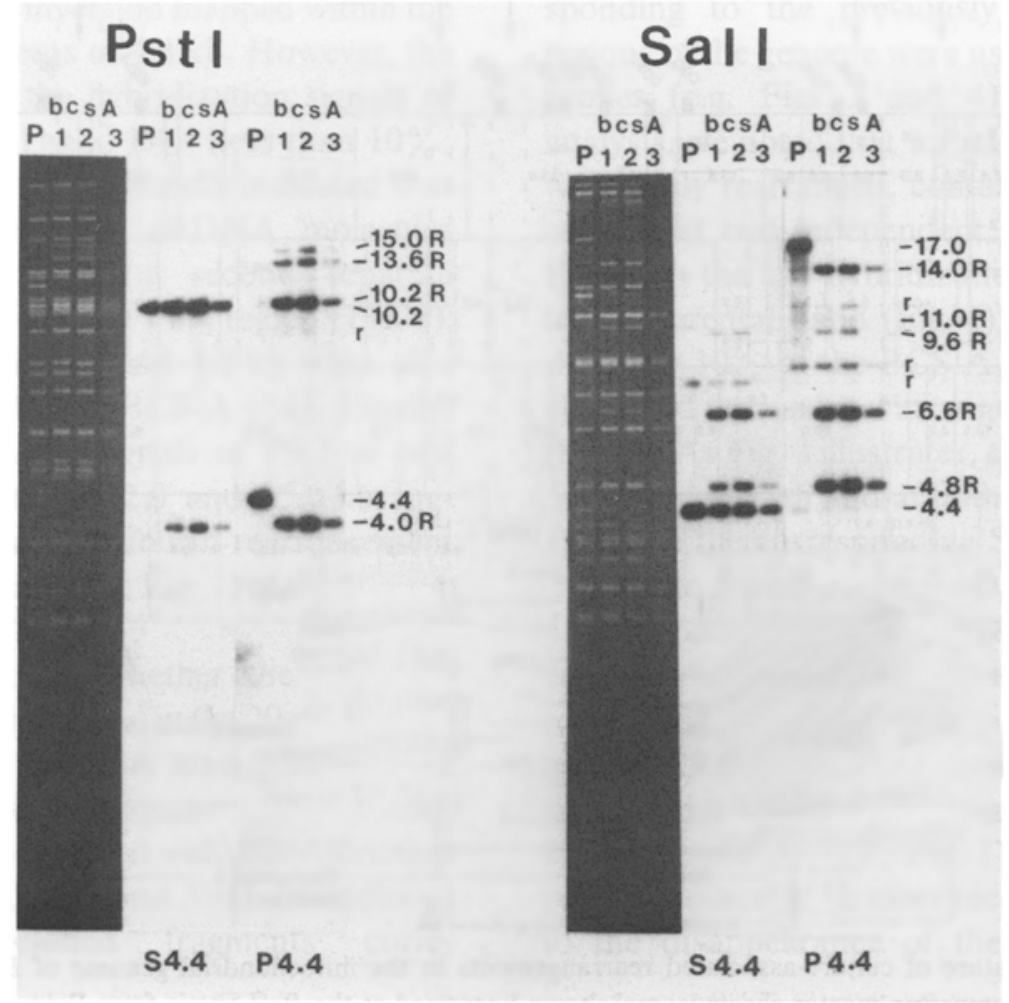

Fig. 3. On-going alteration of mtDNA in culture. MtDNAs from whole plants (P) and cultured cells (BCS-A) were isolated 37 months (bcsA1), 44 months (bcsA2), and 53 months (bcsA3) after initiating the calli, restricted with PstI and $S a l I$, electrophoresed in a $0.7 \%$ agarose gel, transferred to a Zetabind membrane, and hybridized with the indicated cloned fragments. All other labels are as in Fig. 1.

an inversion involving the $\mathrm{S} 10.1$ and $\mathrm{P} 12.2$ regions were reported previously [54] to be present in approximately $30 \%$ of mtDNA molecules (Figs. 1 and 2). With continued growth of BCS-A in culture, the proportion of these novel fragments diminished to less than $10 \%$, whereas the proportion of the parental arrangement of these fragments reached almost that of the control plant (Fig. 4B).

Patterns of mtDNA change in independent culture lines

In order to determine whether the mtDNA changes detected in BCS-A were unique to this culture and whether other $B$. campestris cultures would exhibit no change, the same pattern of change, or different patterns, we analyzed 6 independent cell lines of ages 6 to 11 months (Fig. 5). We also utilized both friable and compact callus cultures to examine whether there is any correlation between callus morphology and the intensity of mtDNA changes in Brassica (Table 1). Figure 5 illustrates $S a l I$ restriction patterns as well as hybridization analysis using the cloned fragment $P 12.2$. With the exception of one culture (X) which revealed a unique alteration, all independent culture lines, regardless of their morphology, contained primarily the same type of rearrangements as BCS-A1 (37-month-old), as evidenced by the appearance of novel, rearranged fragments at 14.0 and $11.0 \mathrm{~kb}$ (Fig. 5). However, the proportion of altered and parental genomes varied greatly among different lines. For example, the somewhat stronger intensity of hybridization of 

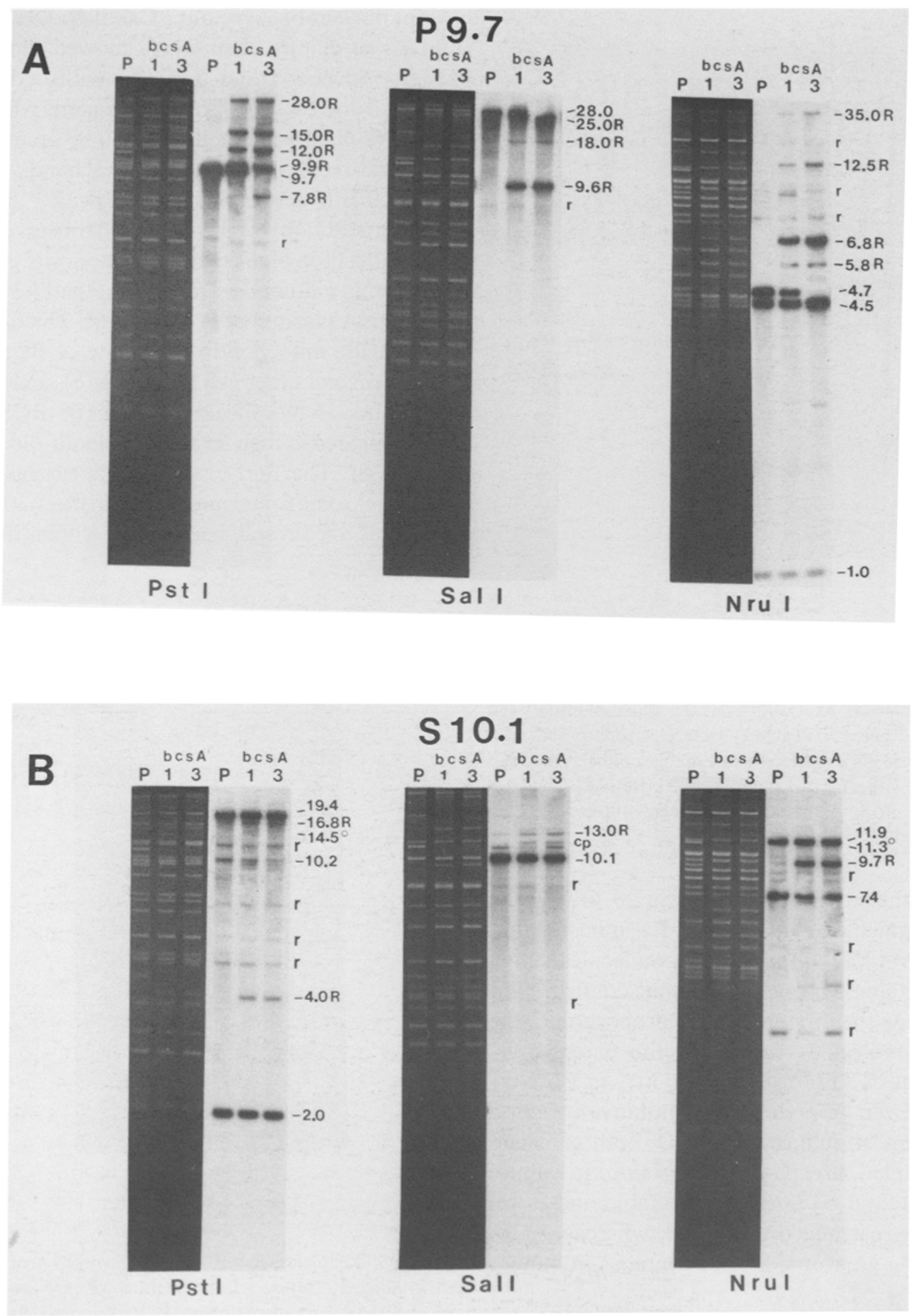

Fig. 4. Hybridization analysis of culture mtDNA alteration over time. mtDNAs from the whole plant (P) and a callus (bscA) were isolated after 37 (bcs-A1) and 53 (bcs-A3) months in culture and hybridized with clones P9.7 (A) and S10.1 (B). The superscript circles $(O)$ denote the disappearance of culture-associated novel fragments previously observed in the mitochondrial genome of BCS-A [54]. All other labels are as in Fig. 1. 


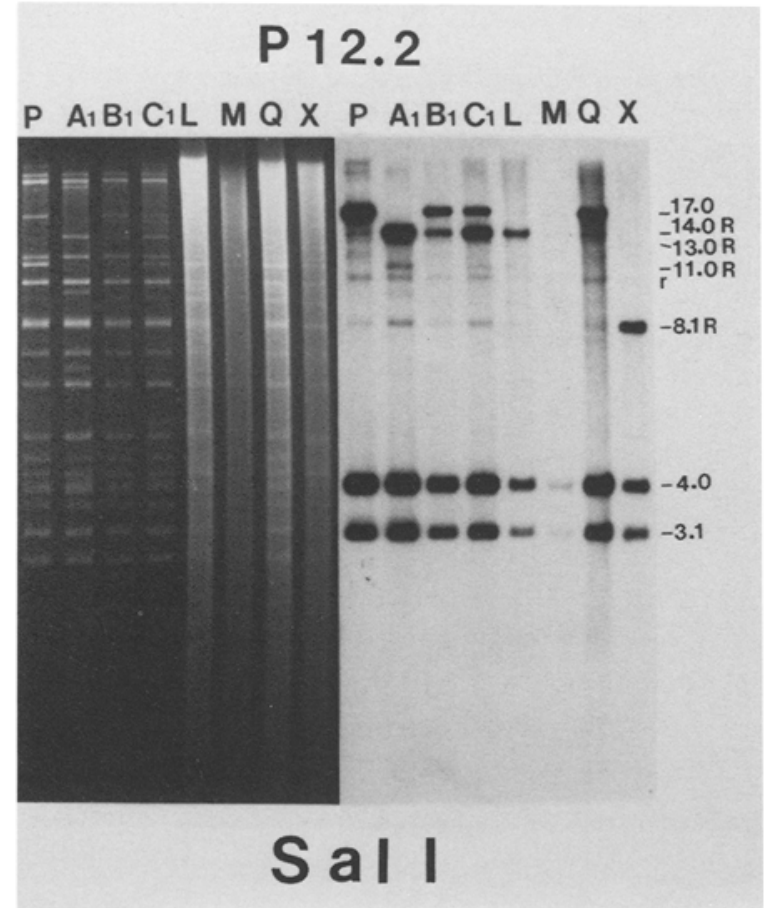

Fig. 5. Instability of mtDNA in long- and short-term cultures. Sal I-restricted mtDNA (P, A, B, C) and total genomic DNA samples $(\mathrm{L}, \mathrm{M}, \mathrm{Q}, \mathrm{X})$ from the whole plant $(\mathrm{P})$ and various cell cultures (Table 1) were electrophoresed on a $0.7 \%$ agarose gel, transferred to Zetabind membranes, and hybridized with a Pst I fragment of $12.2 \mathrm{~kb}$ (P12.2). The sizes of parental and novel hybridizing fragments ( $R$ ) are indicated in $\mathrm{kb}$. All other labels are as in Fig. 1.

P12.2 to the parental fragment, S17.0, than to the novel fragment, S14.0, in BCS-B1 indicates that slightly over half of the mtDNA molecules in this culture retain their original genomic configuration (Fig. 5). On the other hand, the proportion of the mtDNA molecules containing the parental arrangement of $\mathrm{S} 17.0$ is less than $30 \%$ in $\mathrm{BCS}-\mathrm{C} 1$ (Fig. 5). Even more drastic quantitative variation is apparent in cultures $\mathrm{L}$ and $\mathrm{Q}$, both of which were sampled after 11 months in vitro. In culture $\mathrm{L}$ the parental S17.0 is undetectable and is replaced by rearranged fragments, whereas it persists in the great majority of genomes in culture line $Q$ (Fig. 5).

The case of qualitatively new rearranged molecules arising in an independent culture occurred in culture $X$, which was established from another $B$. campestris line (atrazine-resistant) with a differ- ent nuclear background ('Candle'). Detailed analysis of culture X mtDNA showed that a novel large inversion had occurred with endpoints in P12.2 and S4.0. These two fragments which in the native plant genome lie over $80 \mathrm{~kb}$ apart (Fig. 2), hybridized to the same two novel bands, S8.1 and S13.0 (Fig. 5 and data not shown).

In addition to the variation observed in the organization of the main mitochondrial genome of different cultures, we found that the $11.3 \mathrm{~kb}$ linear mtDNA plasmid of Brassica [46], which is absent from the mitochondrial genome of BCS-A [54] and several other cell lines (Fig. 6), including the 6- and 15-month-old cultures of BCS-C, has reappeared in high level in 16-month-old BCS-C3 (Fig. 6). The fairly random appearance of this extrachromosomal molecule in the native plant tissues of several accessions within the genus

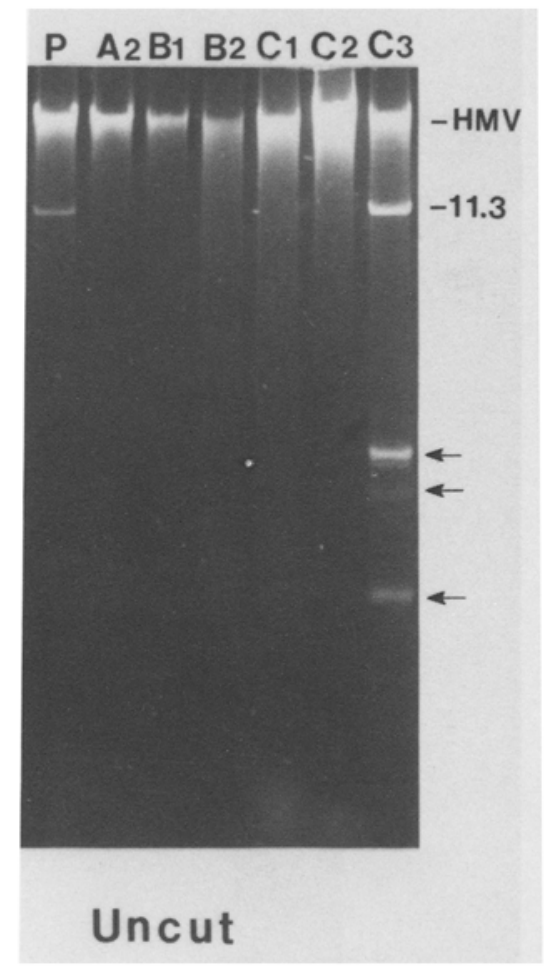

Fig. 6. Reappearance of an $11.3 \mathrm{~kb}$ mtDNA plasmid after prolonged culture. Fractionation of DNase-I purified mtDNAs from whole plant (P) and various Brassica campestris culture lines (Table 1 ) in a $0.7 \%$ agarose gel. HMV denotes high molecular weight undigested mtDNA. The arrows denote the array of putative RNA molecules that occasionally co-purify with mtDNA [24]. 
Brassica has already been demonstrated [46, 24]. The disappearance of this $11.3 \mathrm{~kb}$ plasmid via in vitro culture was also recently shown by Erickson et al. [13]. In their studies, the plasmid was subsequently restored upon sexual hybridization of the regenerants lacking this plasmid and plasmid-containing $B$. napus male plants.

\section{Effect of culture type on mtDNA rearrangement}

To determine whether the same type of alteration would occur in suspension cultures as in callus cultures, we examined mtDNAs of BCS-A and BCS-B cells maintained as suspensions for 12 months (As and Bs, Fig. 7). Hybridization analysis utilizing cloned fragments that correspond to previously identified altered regions of BCS-A (e.g. S4.4, S10.1, P12.2, P4.4; Fig. 1) demon- strated that the mtDNA organization of cells grown as suspensions and callus are generally similar, containing a comparable level of the same type of rearrangements (Fig. 7 and data not shown). The mtDNA profile of the Bs suspension culture appears almost identical to that of the parental callus culture, BCS-B, indicating a lower level of alteration of some regions (e.g. S4.4, Fig. 7) in this cell line, regardless of the culture type. The only differences between the two systems were the most recent rearrangements (e.g. P4.0, S5.0, N9.5) appearing in the BCS-A2 maintained as callus (Fig. 3 and 7) and the rise of a novel molecular species ( $4.6 \mathrm{~kb}$ in size) in the mtDNA of the suspension cultures (Fig. 7). With the exception of $B g l I$ (data not shown), none of the restriction enzymes utilized in this investigation could cleave this $4.6 \mathrm{~kb}$ molecule (Fig. 7). More importantly, this DNA species migrated

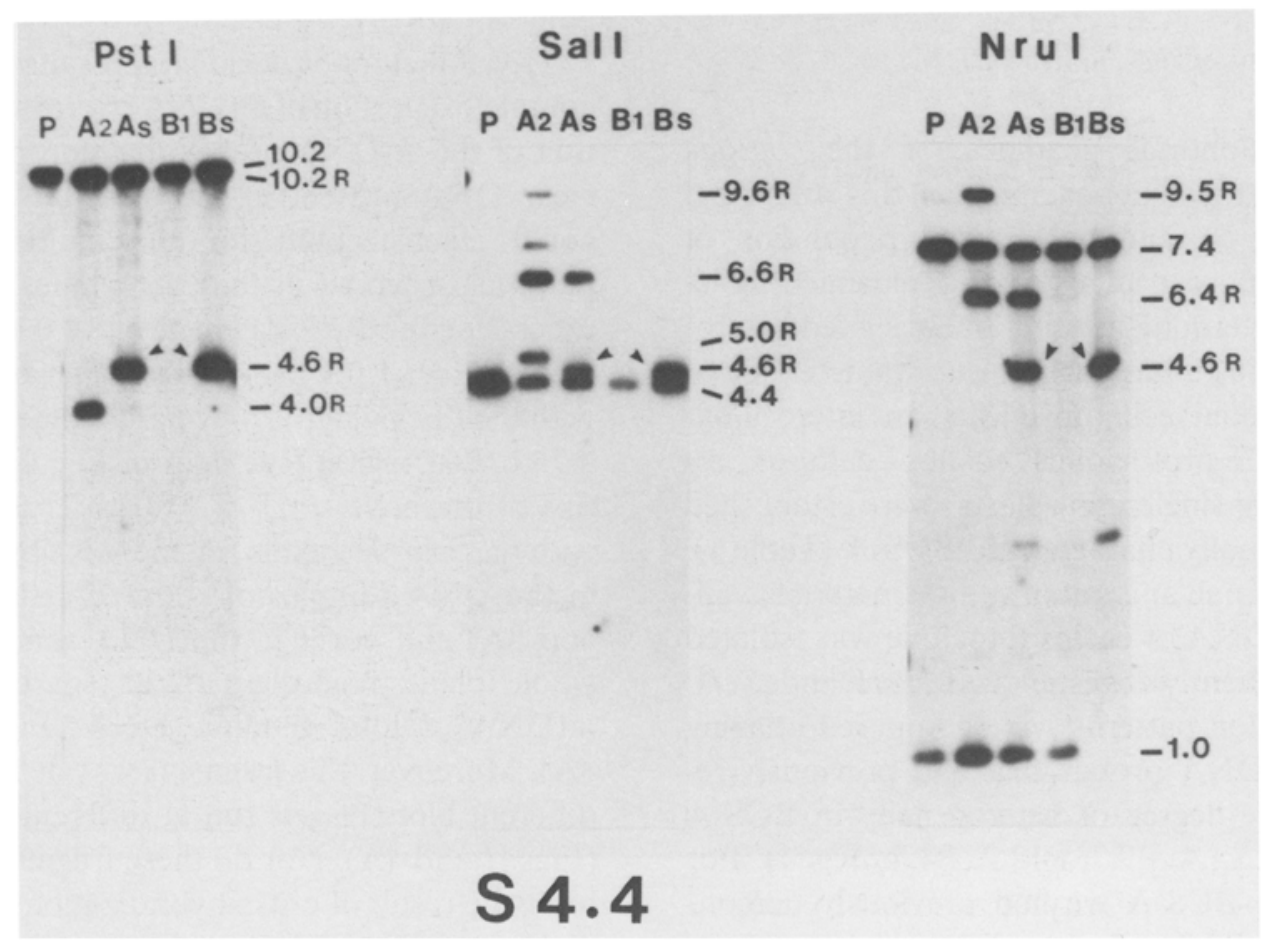

Fig. 7. Comparison of mtDNA organization of callus and suspension cultures. Hybridization of a Sal I fragment of $4.4 \mathrm{~kb}$ (S4.4) to Southern blots of mtDNA samples prepared from whole plant (P), callus cultures (A2, B1), and cell suspensions (As, Bs). The arrowheads indicate a $4.6 \mathrm{~kb}$ plasmid-like molecule present in As and Bs lanes. The absence of the parental NruI fragment of $1.0 \mathrm{~kb}$ in the Bs lane is a blotting artefact since the band was present in other transfers as well as in longer autoradiographic exposure of the same blot. All other labels are as in Fig. 1. 
also as a $4.6 \mathrm{~kb}$ linear molecule in the gels of undigested mtDNA samples of both suspension cell cultures (data not shown) suggesting that this novel species is in fact an extrachromosomal plasmid. However, scarcity of the DNA samples did not permit the determination of whether the $4.6 \mathrm{~kb}$ plasmid was a linear molecule or a circular molecule of some other size. Hybridization analysis using clones from various parts of the mitochondrial genome (e.g. S4.4, S10.1, P12.2, P4.4; S6.2) revealed some degree of sequence similarity between this extrachromosomal plasmid and the chromosomal mtDNA of B. campestris (Fig. 5 and data not shown). A possible explanation for this observation is the presence of a repeat family on the plasmid molecule that is also widely dispersed on the mtDNA chromosome. Perhaps the plasmid originated by recombination between directly oriented repeats flanking an origin of replication on the master chromosome.

\section{Heterogeneity of the culture $m t D N A$}

The mitochondrial genomes of the various Brassica cultured lines examined in this study (e.g. Figs. 3 and 5) contain a mixed population of plant-like ('unrearranged') and rearranged molecules. To determine whether the observed heterogeneity of the culture mtDNA is characteristic of each individual cell or reflects an intercellular variation, 16 protoclones (callus cultures recovered from single protoplasts) were established from the initially characterized BCS-A (Table 1). Due to the small amount of culture material available, total DNA of each protoclone was isolated and subsequently digested with Pst I and SalI. The restriction patterns were compared utilizing specific mtDNA probes that had previously revealed some degree of heterogeneity in BCS-A (e.g. S10.1, S4.4, P9.7, P12.2, P4.4; Fig. 1). For example, in BCS-A we had previously demonstrated that $\mathrm{P} 9.7$ was altered in only $10 \%$ of the mtDNA molecules (Fig. 1). If this was the result of an intercellular heterogeneity $(10 \%$ of the cells having entirely rearranged genomes and the remainder unrearranged ones), then we would ex- pect to find an intact $\mathrm{P} 9.7$ in $90 \%$ of the protoclones. On the contrary, almost all of the protoclone mtDNAs examined here contained some level of culture-associated rearrangements (Fig. 8A). The only exception was P-6, which failed to display any of the rearrangements found in BCS-A. Essentially the same results were obtained using other cloned fragments, including S10.1, S4.4, P12.2, P4.4 and S6.2 (data not shown). Like the parental culture BCS-A, the mtDNA sample of each individual cell line displays a multi-banding pattern, implying the existence of a heteroplasmic state of the mitochondrial genome. The relative intensities of bands within a protoclone mtDNA, however, varied greatly among different protoclones suggesting that the proportion of the rearranged vs. unrearranged genomic types also varies among different cell lines (Fig. 8A). For example, the $12.0 \mathrm{~kb}$ novel fragment is undetectable in the mtDNA of P-37, but quite abundant in the adjacent lane, P-38 (Fig. 8A).

Trivial factors or accidental results such as incomplete digestion of mtDNA or cross-hybridization of the mtDNA probes to chloroplast or nuclear DNA present in the total DNA samples could also account for the multiple-banding patterns observed in the protoclones. However, except for the Pst I fragment of $12.0 \mathrm{~kb}$, none of the novel Pst I fragments are of a size consistent with their being derived by partial digestion of the $9.7 \mathrm{~kb}$ Pst I region (see map in Fig. 2). Even the rise of the novel Pst I of $12.0 \mathrm{~kb}$ is inconsistent with partials, since this fragment is observed only in the DNA samples of cultured cells (Figs. 4A and $8 \mathrm{~A}$ ) and never in the DNA samples of the whole plant, regardless of its source (purified mtDNA or total genomic DNA; Figs. 4A and $8 \mathrm{~A})$. Moreover, this fragment was detected using different blots of gels run at different timepoints (Figs. 1 and 4A). Nor do these fragments appear to be the result of cross-hybridizations to chloroplast or nuclear DNA; previous studies [40] have demonstrated no detectable homology between P9.7 and cpDNA of Brassica. Furthermore, probing purified mtDNA samples of cultured cells revealed patterns identical to those observed 

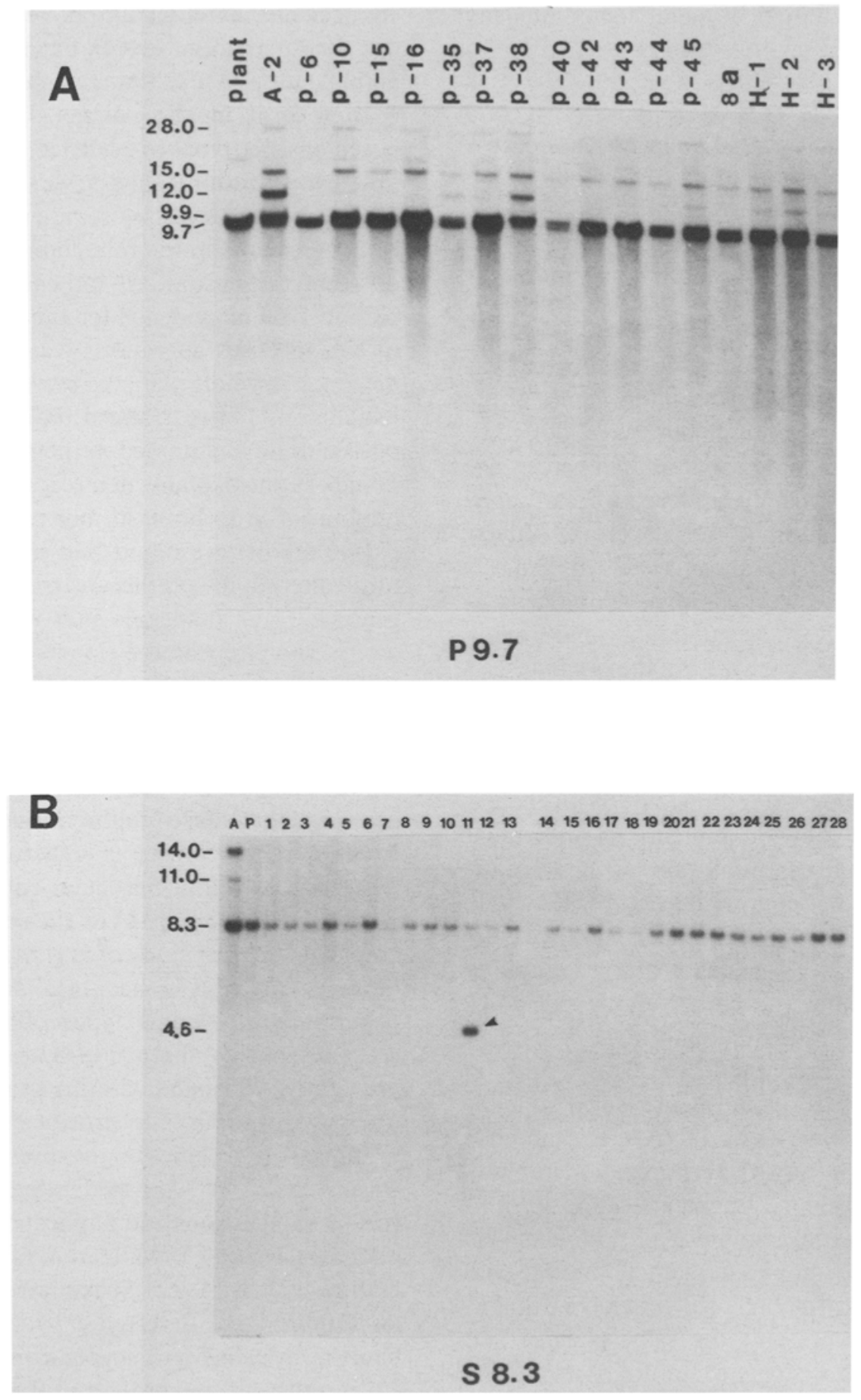

Fig. 8. Heterogeneity of mtDNA from protoclones and individual plants. A. A Pst I fragment of $9.7 \mathrm{~kb}$ (P9.7) was hybridized to $P s t$ I digests of $B$. campestris total genomic DNA isolated from a bulk plant population $(\mathrm{P})$, a callus culture (A-2) and various protoclones (Table 1). B. A SalI fragment of $8.3 \mathrm{~kb}$ (S8.3) was hybridized to total genomic DNAs of 28 rapid-cycling plants from the same seed batch used to initiate various cultures used in this study ( 1 through 28 ). The arrow denotes a $4.6 \mathrm{~kb}$ plasmid-like molecule in lane 11. Numbers to the left of each panel refer to the size of parental (plant-like) and culture-associated restriction fragments. 
in protoclones, although at much higher intensity (see for example Fig. 4A).

Heterogeneity of the mtDNA among B. campestris plants

The variable proportions of parental and rearranged mtDNA molecules in different cultures could theoretically have originated from a heterogeneous seed lot containing a mixed population of mitochondrial genomes. Although a high level of culture-associated rearrangements at the whole plant level was originally ruled out [54], there still existed a chance that a small percentage of seeds contained significant levels of rearranged genome forms, but that these were effectively masked by a predominance of seeds with entirely unrearranged molecules. To investigate this possibility, total DNA of 28 individual rapid-cycling $B$. campestris plants grown from the original seed stock (the source of all culture lines) was restricted with PstI (data not shown) and SalI (Fig. 8B) and subsequently probed with mtDNA clones representing altered regions. With the exception of one individual plant, all individual plants exhibited an essentially unrearranged mitochondrial genome comparable to bulk whole plant mtDNA (Fig. 8B). The only significant exception was plant 11 , which featured a unique band of approximately $4.6 \mathrm{~kb}$. Side-by-side comparison of the undigested and Pst $\mathrm{I}$ and SalI restricted DNAs prepared from plant 11 and the suspension cultures As and Bs, revealed an identical mobility for this $4.6 \mathrm{~kb}$ DNA species in both systems (data not shown). Hybridization analysis utilizing various cloned fragments of the $B$. campestris mitochondrial genome against blots of gels containing DNA samples of plant 11 and the suspension cultures provided further evidence that in fact this band represents the same plasmid-like molecule in both systems (data not shown).

\section{Discussion}

Our analyses of a wide range of $B$. campestris culture lines illustrate that rapid structural changes of the mitochondrial genome in vitro are not due to random events but rather reflect the amplification of a pre-existing pool of rearranged genome forms in whole plants. This conclusion is based on three types of evidence. First, by detailed filter hybridization analysis, we were able to identify varying levels of identical structural rearrangements in the mitochondrial genomes of different suspension cell and callus cultures. The second type of evidence for the non-random nature of mtDNA alterations was provided by the detection in whole plants of low abundance forms ('sublimons') of rearranged molecules which appeared to be maintained in the mitochondrial genome of control plants at a recombinational equilibrium. Third, both in our evolutionary and in vitro studies we noted that most of the structural alterations of the Brassica mitochondrial genome occur at specific sites via recombination across short repeated elements.

Although a number of studies have already demonstrated an increased plasticity of plant mtDNA in response to tissue culture manipulations $[17,7,39,41,50,21,4]$ it is still uncertain as to whether these 'culture-induced' alterations occur systematically in all higher-plant species or whether they reflect particular culture conditions such as culture age [34] or the in vitro technique employed [23]. A study of in vitro mtDNA alterations of maize [7] has also shown that the occurrence of specific types of rearrangements (i.e. presence or absence of S1 and S2 plasmid) are tightly associated with the friable callus morphology. Both in our previous work (see for example Fig. 1) and in the present study (e.g. Figs. 3 and 4A), we noted extensive alterations (i.e. large inversions and duplications) in the mitochondrial genome organization of a $B$. campestris culture line (BCS-A). Strikingly similar types of alterations were detected in various long- and short-term callus and suspension cell lines (6 to 11 months old), regardless of the type and morphology of the culture (Fig.5). A qualitatively distinct type of alteration was observed in the mitochondrial genome configuration of one culture line (X), which was initiated from a different B. campestris cultivar (Candle). Further analysis 
of the mtDNA of this culture line showed that a novel rearrangement (i.e. inversion) with endpoints in P12.2 and S4.0 is responsible for the observed variation (Fig. 3 and data not shown). Interestingly, the endpoints of an evolutionary inversion distinguishing the mitochondrial genome organization of $\boldsymbol{B}$. campestris and $\boldsymbol{B}$. oleracea have also been mapped within these same regions [45]. These results imply that the apparent instability of $B$. campestris mtDNA was not an isolated event unique to the initially characterized cell line, BCS-A. Our analysis of another species, Raphanus sativa (Ogura cytoplasm in B. campestris nuclear background), following six months of in vitro growth provided further evidence for the general instability of the mitochondrial genome of different members of Brassicaceae. Our preliminary results indicate at least alterations mapping within a SalI fragment of $11.8 \mathrm{~kb}$ [see map in 33] as well as sequences flanking atp9, atp6, and coxl regions (unpublished data). The susceptibility of the Ogura mitochondrial genome to structural changes following in vitro manipulations has also been reported by other groups employing different techniques [47, 36, 35, 21].

Regarding the impact of the age of culture on the degree of mtDNA rearrangement, in two recent studies of embryogenic wheat cultures, Hartmann et al. [20] and Rode et al. [50] concluded that most changes of the mitochondrial genome in vitro occur during the early process of callogenesis and that these changes are conserved in later subcultures. However, in our study with various non-embryogenic $B$. campestris cultured cells, we found no evidence that structural changes of mtDNA were limited to any particular timepoint, nor did we find that all changes were necessarily maintained in subsequent generations. For example, periodic sampling of mtDNA of BCS-A for five consecutive years demonstrated that, with continued growth in culture, altered mtDNA molecules continued to arise (e.g. P4.0 and S.4.8; Fig. 3), as well as to both increase (e.g. P9.9, P16.8; Fig. 4A) and diminish in intensity (e.g. P14.5, S13.0; Fig. 4B). Therefore, based on these observations and similar reports $[32,6,12]$, it appears that in most but not all cases $[20,50]$ the duration of in vitro growth has a continuing impact on the level of changes of the plant mitochondrial genome. At present, there is little evidence for the effect of culture systems (e.g. type, morphology, technique, or age) on the extent of mtDNA alteration in Brassica. Whether the nuclear background $[7,2,34]$ could have an impact on the rapid structural changes of mitochondrial genome in vitro is yet to be determined.

Is the appearance of 'novel' mtDNA molecules in cultured plant cells the outcome of de novo recombination and rearrangement, or does it simply represent an amplification of pre-existing low abundance molecules? Evidence in support of the latter model has already been provided by Small et al. [56, 57] who examined the maize atp $A$ gene region. In their studies of various fertile (N) and sterile (cms) maize mtDNAs, they were able to detect in fertile lines atp $A$ sequences, previously believed to be unique to sterile cytoplasms, but at sub-stoichiometric level ('sublimons'). Considering that these molecules were retained through many generations at extremely low abundance, Small et al. [56] ruled out continuous de novo recombination as the primary source of these sublimons. Similarly, in our studies of $B$. campestris cultured cells ([54] and Fig. 1) we noted that traces of some of the cultureinduced alterations existed already in the mtDNA sample of the control plants (see for example Fig. 1). This observation strongly implies that most, if not all, of the existing variation between whole plant and cultured cell mtDNA of $B$. campestris could be due to differences in the stoichiometry of existing forms rather than de novo formation of novel restriction fragments. Therefore, it is quite conceivable that the primary effect of tissue culture manipulation on the organization of the $B$. campestris mitochondrial genome is limited to an enhancement of pre-existing heterogeneity in the parental plant genome. In fact, an enhanced state of heteroplasmy is quite evident in the mtDNA profile of various shortand long-term culture lines, all of which reveal various ratios of 'parental' (more abundant in plant) to 'novel' (less abundant in plant) forms of the genome (e.g. Figs. 1, 4 and 5). 
To investigate whether the apparent heterogeneity is characteristic of each individual cell or reflects an intercellular variation, 16 clones derived from single protoplasts of the 43-monthold callus culture BCS-A were compared. A mixed population of unrearranged and rearranged mtDNA molecules was apparent in each protoclone (see Fig. 8A), suggesting that the observed heterogeneity perhaps reflects the genomic composition of each individual cell. However, we can not completely rule out the possibility of a genotypic variation in the cell population subsequent to the protoplast isolation. The heterogeneous mitochondrial genome at the whole plant level could have theoretically resulted from a mixed population of plant-like and culture-like cytoplasms within the parental seed source. However, our comparison of mtDNA patterns of 28 individual plants to that of the pooled control plants failed to show any significant variation in the level of culture-induced alterations among individual plants. Therefore, source plant variation is unlikely to have contributed to the diversity of mitochondrial genomes observed in cultured cells. The only exception to this general phenomenon was the rise of a plasmid-like molecule of $4.6 \mathrm{~kb}$ in size, which appeared in equimolar intensity in the mitochondrial genomes of two independently established suspension cell lines as well as in one individual plant (see Figs. 7 and 8B). Although it is possible that the independent suspension cell cultures were both fortuitously derived from hypocotyls with a genotype similar to that of the plant 11, such an occurrence is unlikely, since only one out of 28 plants displayed a high level of this molecule. An alternative explanation is that traces of the $4.6 \mathrm{~kb}$ molecule pre-existed in the mitochondrial genome of parental callus cultures (BCS-A and BCS-B) as well as in other individual plants, but for reasons as yet unknown, the molecule was selectively amplified and sorted out in a single plant and two suspension lines. In other species following somatic hybridization, preferential amplification of pre-existing substoichiometric molecules has also been suggested to explain the occurrence of novel restriction fragments not representative of either parent $[37,42]$.
These observations together with other reports [see for review 28, 29] strongly imply that the plant mitochondrial genome is a dynamic system composed of various genomic types which coexist at different copy numbers in a state of recombinational equilibrium. It is unclear why in cultured cells and occasionally in organized plant tissues the control on this equilibrium is lost, leading to a selective amplification and reassortment of the previously low abundance forms. Although no study has yet shown the direct impact of in vitro stress on mitochondrial configuration, there are a few reports showing significant variation in number, size and morphology of mitochondria in various organized plant tissues [ 1 , $11,61]$ and in several suspension and callus cultures [52, 59]. For example, after seven months of in vitro growth, Ginkgo biloba cultured cells contained an increased number of small mitochondria dispersed throughout the cytoplasm, as well as an increase in the density of mitochondrial stroma [51, 52]. Such an increase in the number of mitochondria per cell (from 110-140 per apical cell to 1000 in cotyledon cells or perhaps greater in cultured cells), could result in the average number of genomes per cell becoming less than the average number of mitochondria per cell. Whether or not the formation of partial genomes in individual mitochondria and possible variation in the proportion of mitochondria containing a particular low abundance genome form (e.g. sublimon) is the source of the observed mtDNA variation in $B$. campestris cultures will require further investigation.

Regardless of the source of mtDNA variability in higher plants, the final stoichiometry of each genomic type will depend on the rate of homologous recombination [for review see 29,4 ], which is in turn determined by the size and sequence composition of repeated elements [63]. In the case of large repeats (greater than $1 \mathrm{~kb}$ in size), high-frequency recombination results in nearly equimolar production of recombination isomers $[44,30,55]$. Occasionally, however, infrequent recombination events across minor repeats might generate low-abundance molecules which are retained through many plant generations [ 56,57$]$. 
In fact, in our evolutionary studies of the Brassica mitochondrial genome (unpublished data), we have been able to identify over 23 such minor repeat families, ranging from 100 to 450 base pairs in length. Furthermore, certain regions of the mitochondrial genome (e.g. S10.1, P12.2, P4.4), which in this investigation and in evolutionary studies of the genus Brassica [45] appeared especially susceptible to structural mutations, were found to contain two to three independent repeat families. Therefore, the location and prevalence of these repeated elements suggest infrequent homologous recombination as the possible underlying mechanism for the generation of the observed heterogeneity in mtDNA of $B$. campestris plants and cultured cells.

\section{Acknowledgments}

We thank T. Deussen for assistance in typing and editing the manuscript and $\mathrm{C}$. Condit and J. Seemann for critical reading of the manuscript. This research was supported by NIH grant GM35087 to J.D.P, USDA grant 85-00363 to E.D.E, and a NRAC postgraduate fellowship from the New Zealand government to M.C.

\section{References}

1. Bendich AJ, Gauriloff LP: Morphometric analysis of cucurbit mitochondria: the relationship between chondriome volume and DNA content. Protoplasma 119: 1-7 (1984).

2. Bork K, Walbott V: Comparison of the RE digestion patterns of mtDNA from normal and CMS of Zea mays. Genetics 102: 109-128 (1982).

3. Brears $\mathrm{T}$, Lonsdale DM: The sugarbeet $\mathrm{mt}$ genome: a complex organization generated by homologous recombination. Mol Gen Genet 214: 514-522 (1988).

4. Brears T, Curtis GJ, Lonsdale DM: A specific rearrangement of mitochondrial DNA induced by tissue culture. Theor Appl Genet 77: 620-624 (1989).

5. Brettell RIS, Thomas E, Ingram DS: Reversion of Texas male-sterile cytoplasm maize in culture to give fertile T-toxin resistant plants. Theor Appl Genet 58: 55-58 (1980).

6. Buyser JD, Hartmann C, Henry Y, Rode A: Variations in long-term wheat somatic tissue culture. Can J Bot 66 1891-1895 (1988).
7. Chourey PS, Kemble RJ: Transposition events in tissue cultured cells of maize. In: Fujiwara A (ed) Plant tissue culture 1982, pp 425-426. Japanese Association for Plant Tissue Culture, Tokyo (1982).

8. Dale RMK, Duesing JH, Keen D: Supercoiled mitochondrial DNAs from plant tissue cultured cells. Nucleic Acids Res 9: 4583-4593 (1981).

9. De Francesco L, Attardi G, Croce CM: Uniparental propagation of mitochondrial DNA in mouse-human hybrids. Proc Natl Acad Sci USA 77: 4079-4083 (1980).

10. Doyle JJ, Doyle JL: A rapid DNA isolation procedure for small quantities of fresh leaf tissue. Phytochem Bull 19: 11-15 (1987).

11. Duckett JD, Toth R: Giant mitochondria in a periclinal chimera, Ficus elastica Roxb. Ann Bot 41: 903-912 (1977).

12 Earle ED, Gracen VE, Best VM, Batts LA, Smith ME: Fertile revertants from $S$-type male-sterile maize grown in vitro. Theor Appl Genet 74: 601-609 (1987).

13. Erickson L, Kemble RJ, Swanson E: The Brassica mitochondrial plasmid can be sexually transmitted. Pollen transfer of a cytoplasmic genetic element. Mol Gen Genet 218: 419-422 (1989).

14. Evans DA, Sharp WR: Single gene mutations in tomato plants regenerated from tissue culture. Science 22: 949-951 (1983).

15. Galun E, Arzee-Gonen P, Fluhr R, Edelman M, Aviv D: Cytoplasmic hybridization in Nicotiana: mitochondrial DNA analysis in progenies resulting from fusion between protoplasts having different organelle constitutions. Mol Gen Genet 186: 150-156 (1982).

16. Gengenbach BG, Green CE, Donovan CE: Inheritance of selected pathotoxin resistance in maize plants regenerated from cell cultures. Proc Natl Acad Sci USA 74: 5113-5117 (1977).

17. Gengenbach BG, Connelly JA, Pring DR, Conde MF: Mitochondrial DNA variation in maize plants regenerated during tissue culture selection. Theor Appl Genet 59: 161-167 (1981).

18. Greyburn WS, Bendich AJ: Variable abundance of a mitochondrial DNA fragment in cultured tobacco cells. Curr Genet 12: 257-261 (1987).

19. Hanson MR: Stability, variation, and recombination in plant mitochondrial genomes via tissue culture and somatic hybridisation. Oxford Surv Plant Mol Cell Biol 1: 33-52 (1984).

20. Hartmann C, de Buyser J, Henry Y, Falconet D, Lejeune B, Benslimane A, Quétier F, Rode A: Time-course of mitochondrial genome variation in wheat embryogenic somatic tissue cultures. Plant Sci 53: 191-198 (1987).

21. Jourdan PS, Earle ED, Mutschler MA: Synthesis of male sterile, triazine-resistant Brassica napus by somatic hybridization between cytoplasmic male sterile $B$. oleracea and atrazine resistant B. campestris. Theor Appl Genet 78: 445-455 (1989).

22. Karp A, Bright SWJ: On the causes and origins of somaclonal variations. In: Miflin BJ (ed) Oxford Surveys of 
Plant Molecular Biology, Volume 2, pp. 199-234. Oxford University Press (1985).

23. Kemble RJ, Yarrow SA, Wu SC, Barsby TL: Absence of mitochondrial and chloroplast DNA recombinations in Brassica napus plants regenerated from protoplasts, protoplast fusions and anther culture. Theor Appl Genet $75: 875-881$ (1988).

24. Kemble RJ, Carlson JE, Erickson LR, Sernyk JL, Thompson DJ: The Brassica mitochondrial DNA plasmid and large RNAs are not exclusively associated with cytoplasmic male sterility. Mol Gen Genet 205: 183-185 (1986).

25. Kolodner R, Tewari KK: Physiochemical characterization of mitochondrial DNA from pea leaves. Proc Natl Acad Sci USA 69: 1830-1834 (1972).

26. Larkin PJ, Scowcroft WR: Somaclonal variation - a novel source of variability from cell cultures for plant improvement. Theor Appl Genet 60: 197-214 (1981).

27. Lentini $Z$, Christey M, Earle ED, Mutchler MA: Cell suspension culture of rapid-cycling Brassica campestris $\mathbf{L}$. Cruciferae Newsletter 11: 86-87 (1986).

28. Levings CS III, Brown GG: Molecular biology of plant mitochondria. Cell 56: 171-179 (1989).

29. Lonsdale DM, Brears T, Hodge TP, Melville SE, Rottmann WH: The plant mitochondrial genome: homologous recombination as a mechanism for generating heterogeneity. Phil Trans R Soc Lond B 319: 149-163 (1988).

30. Lonsdale DM, Hodge TP, Fauron CMR, Flavell RB: A predicted structure for the mitochondrial genome from the fertile cytoplasm of maize. In: Goldberg RB (ed) UCLA Symposia on molecular and cellular biology, vol. 12: 445-456. Alan R. Liss, New York (1983).

31. Lörz H, Göbel E, Brown P: Advances in Tissue Culture and Progress Towards Genetic Transformation of Cereals. Plant Breeding 100: 1-25 (1988).

32. Maddock SE, Semple JT: Field assessment of somaclonal variation in wheat. J Exp Bot 37: 1065-1078 (1986).

33. Makaroff CA, Palmer JD: Mitochondrial DNA rearrangements and transcriptional alterations in the male-sterile cytoplasm of Ogura radish. Mol Cell Biol 8: 1474-1480 (1988).

34. McNay JW, Chourey PS, Pring DR: Molecular analysis of genomic stability of mitochondrial DNA in tissue cultured cells of maize. Theor Appl Genet 67: 433-437 (1984).

35. Menczel L, Morgan A, Brown S, Maliga P: Fusion mediated combination of Ogura-type cytoplasmic male sterility with Brassica napus plastids using X-irradiated CMS protoplasts. Plant Cell Report 6: 98-101 (1987).

36. Morgan A, Maliga P: Rapid chloroplast segregation and recombination of mitochondrial DNA in Brassica cybrids. Mol Gen Genet 209: 240-246 (1987).

37. Morgens PH, Grabau EA, Gesteland RF: A novel soybean mitochondrial transcript resulting from a DNA rearrangement involving the $5 \mathrm{~S}$ rRNA gene. Nucl Acids Res 12: 5665-5684 (1984).

38. Nagy F, Lazar G, Menczel L, Maliga P: A heteroplasmic state induced by protoplast fusion is a necessary condition for detecting rearrangements in Nicotiana mitochondrial DNA. Theor Appl Genet 66: 203-207 (1983).

39. Negruk VI, Eisner GI, Redichkina TD, Dumanskaya NN, Cherny DI, Alexandrov AA, Shemyakin MF, Butenko RG: Diversity of Vicia faba circular mtDNA in whole plants and suspension cultures. Theor Appl Genet 72: 541-547 (1986).

40. Nugent JM, Palmer JD: Location, identity, amount and serial entry of chloroplast DNA sequences in crucifer mitochondrial DNAs. Curr Genet 14: 501 (1988).

41. Ozias-Akins P, Pring DR, Vasil IK: Rearrangement in the mitochondrial genome of somatic hybrid cell lines of Pennisetum americanum (L.) K.Schum + Panicum maximum Jacq. Theor Appl Genet 74: 15-20 (1987).

42. Ozias-Akins $P$, Tabaeizadeh $Z$, Pring DR, Vasil IK: Preferential amplification of mitochondrial DNA fragments in somatic hybrids of the Graminae. Curr Genet 13: 241-245 (1988).

43. Palmer JD: Isolation and structural analysis of chloroplast DNA. Meth Enzymol 118: 167-186 (1986).

44. Palmer JD, Shields CR: Tripartite structure of the Brassica campestris mitochondrial genome. Nature 307 : 437-440 (1984).

45. Palmer JD, Herbon LA: Plant mitochondrial DNA evolves rapidly in structure, but slowly in sequence. J Mol Evol 28: 87-97 (1988).

46. Palmer JD, Shields CR, Cohen DB, Orton TJ: An unusual mitochondrial DNA plasmid in the genus Brassica. Nature 301: 725-728 (1983).

47. Pelletier G, Primard C, Vedel F, Chetrit P, Remy R, Rouselle $\mathrm{P}$, Renard M: Intergeneric cytoplasmic hybridization in Cruciferae by protoplast fusion. Mol Gen Genet 191: 244-250 (1983).

48. Pring DR, Lonsdale DM, Gracen VE, Smith AG: Mitochondrial DNA duplication/deletion events and polymorphisms of the $\mathrm{C}$ group of the male sterile cytoplasms. Theor Appl Genet 73: 646-653 (1987).

49. Robertson D, Earle ED: Plant regeneration from leaf protoplasts of Brassica oleracea var italica cv Green Comet broccoli. Plant Cell Rep 5: 61-64 (1986).

50. Rode A, Hartmann C, Falconet D, Lejeune B, Quétier F, Benslimane A, Henry Y, de Buyser J: Extensive mitochondrial DNA variation in somatic tissue cultures initiated from wheat immature embryos. Curr Genet 12: 369-376 (1987).

51. Rohr R: Existence d'un reticulum mitochondrial dans les cellules d'une culture de tissu haploide d'une plante vasculaire. Biol Cellulaire 33: 89-92 (1978).

52. Rohr R: Reconstruction tridimensionelle de chondriome de Ginko en culture tissulaire; étude au moyen de coupes épaisses et de coupes fine sériées. Can J Bot 57 : 332-340 (1979). 
53. Rottman WH, Brears R, Hodge TP, Lonsdale DM: A mitochondrial gene is lost via homologous recombination during reversion of cms-T maize to fertility. EMBO J 6: 1541-1546 (1987).

54. Shirzadegan M, Christey M, Earle ED, Palmer JD: Rearrangement, amplification, and assortment of mtDNA molecules in cultured cells of Brassica campestris. Theor Appl Genet 77: 17-25 (1989).

55. Siculella L, Palmer JD: Physical and gene organization of mitochondrial DNA in fertile and CMS sunflower. CMS associated alterations in structure and transcription of atpA gene. Nucl Acids Res 16: 3787-3799 (1988).

56. Small ID, Isaac PG, Leaver CJ: Stoichiometric differences in DNA molecules containing atpA suggest mechanisms for the generation of mitochondrial genome diversity in maize. EMBO J 6: 865-869 (1987).

57. Small ID, Suffolk R, Leaver CJ: Evolution of plant mitochondrial genomes via substoichiometric intermediates. Cell 58: 69-76 (1989).

58. Smith AG, Chourey PS, Pring DR: Replication and amplification of the small mitochondrial DNAs in a cell suspension of Black Mexican Sweet maize. Plant Mol Biol 10: 83-90 (1987).

59. Thomas MR, Rose RJ: Plastid number and plastid structural changes associated with tobacco mesophyll protoplast culture and plant regeneration. Planta 158: 329-338 (1983).

60. Vedel F, Chetrit P, Mathieu C, Pelletier G, Primard C: Several different mitochondrial DNA regions are involved in intergenomic recombination in Brassica napus cybrid plants. Curr Genet 11: 17-24 (1986).

61. Walbot V, Thompson D, Veith GM, Coe EH Jr: Nuclear genes controlling chloroplast development. In: Leaver CJ (ed.) Genome Organization and Expression in Plants, pp. 381-399. Plenum. New York (1980).

62. Wilson AJ, Chourey PS, Sharpe DZ; Protoclones of Sorghum bicolor with unusually high mitochondrial DNA variation. In: Henke RR, Hughes KW, Constantin MJ, Hollaender A (eds) Tissue culture in forestry and agriculture, pp 368-369 (1985).

63. de Zamaroczy M, Faugeron-Fonty G, Bernardi G: Excision sequences in the mitochondrial genome of yeast. Gene 21: 193-202 (1983). 